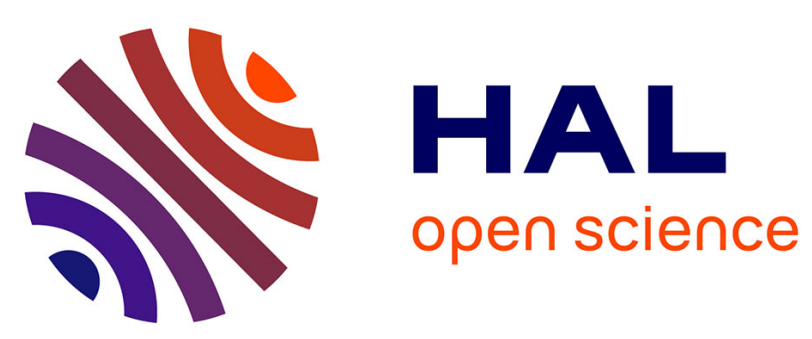

\title{
Emery-Dreifuss muscular dystrophy: focal point nuclear envelope
}

\author{
Antoine Muchir, Howard J Worman
}

\section{To cite this version:}

Antoine Muchir, Howard J Worman. Emery-Dreifuss muscular dystrophy: focal point nuclear envelope. Current Opinion in Neurology, 2019, 32 (5), pp.728 - 734. 10.1097/wco.0000000000000741. hal-03463390

\section{HAL Id: hal-03463390 \\ https://hal.sorbonne-universite.fr/hal-03463390}

Submitted on 2 Dec 2021

HAL is a multi-disciplinary open access archive for the deposit and dissemination of scientific research documents, whether they are published or not. The documents may come from teaching and research institutions in France or abroad, or from public or private research centers.
L'archive ouverte pluridisciplinaire HAL, est destinée au dépôt et à la diffusion de documents scientifiques de niveau recherche, publiés ou non, émanant des établissements d'enseignement et de recherche français ou étrangers, des laboratoires publics ou privés. 


\title{
Emery-Dreifuss muscular dystrophy: focal point nuclear envelope
}

\author{
Antoine Muchir ${ }^{\mathrm{a}}$ and Howard J. Worman ${ }^{\mathrm{b}, \mathrm{c}}$
}

\begin{abstract}
Purpose of review
Emery-Dreifuss muscular dystrophy (EDMD) is caused by mutations in EMD encoding emerin and LMNA encoding A-type lamins, proteins of the nuclear envelope. In the past decade, there has been an extraordinary burst of research on the nuclear envelope. Discoveries resulting from this basic research have implications for better understanding the pathogenesis and developing treatments for EDMD.

\section{Recent findings}

Recent clinical research has confirmed that EDMD is one of several overlapping skeletal muscle phenotypes that can result from mutations in EMD and LMNA with dilated cardiomyopathy as a common feature. Basic research on the nuclear envelope has provided new insights into how A-type lamins and emerin function in force transmission throughout the cell, which may be particularly important in striated muscle. Much of the recent research has focused on the heart and LMNA mutations. Prevalence and outcome studies have confirmed the relative severity of cardiac disease. Robust mouse models of EDMD caused by LMNA mutations has allowed for further insight into pathogenic mechanisms and potentially beneficial therapeutic approaches.
\end{abstract}

\section{Summary}

Recent clinical and basic research on EDMD is gradually being translated to clinical practice and possibly novel therapies.

\section{Keywords}

cardiomyopathy, emerin, lamin, muscular dystrophy, nuclear envelope

\section{INTRODUCTION}

In 1966, Emery and Dreifuss reported on a large family from Virginia with X-linked recessive inheritance slowly progressive muscle weakness and wasting in a scapulohumeroperoneal distribution, early contractures of the elbows, ankles, and posterior neck, and cardiac disease [1]. Céstan and LeJonne [2] at l'Hôpital de la Salpêtrière in Paris had described a similar disease phenotype over half a century earlier. Rowland et al. [3] at Columbia University in New York reported an additional case in 1979 and suggested that the unique clinical signs seem to define a distinct form of muscular dystrophy, warranting the designation 'Emery-Dreifuss' type. Subsequent studies demonstrated autosomal dominant inheritance of the same phenotype. More recent research has deciphered the molecular genetics of Emery-Dreifuss muscular dystrophy (EDMD), which has allowed for some progress towards understanding pathogenesis and possible therapies. Here we review some of the recent research relevant to EDMD and attempt to put it into context of what we know about it clinically.

\section{GENOTYPES AND PHENOTYPES: THE SAME AND NOT THE SAME}

EDMD is a genetically heterogeneous condition. In pioneering research, cases of X-linked inheritance were first linked to mutations in $E M D$ encoding emerin [4] and subsequently autosomal dominant inheritance to LMNA encoding A-type nuclear lamins [5]. EDMD-like phenotypes have also been reported in several small studies to result from mutations in genes encoding nesprin-1, nesprin-2, SUN1, SUN2, LUMA, and lamina-associated

\footnotetext{
aSorbonne Université, INSERM UMRS974, Center of Research in Myology, Institut de Myologie, Paris, France, ${ }^{b}$ Department of Medicine and ${ }^{\mathrm{C}}$ Department of Pathology and Cell Biology, Vagelos College of Physicians and Surgeons, Columbia University, New York, New York, USA

Correspondence to Howard J. Worman, MD, Department of Medicine, Vagelos College of Physicians and Surgeons, Columbia University, 630 West 168th Street, New York, NY 10032, USA. Tel: +1 212305 1306; e-mail: hjw14@columbia.edu
}

Curr Opin Neurol 2019, 32:728-734

DOI:10.1097/WCO.0000000000000741 


\section{KEY POINTS}

- EDMD is caused by mutations in genes encoding proteins of the nuclear envelope, with definitive linkages to mutations in EMD and $L M N A$, respectively, encoding emerin and A-type lamins and possible linkages to genes encoding other proteins of this organelle.

- Mutations in these genes may cause muscular dystrophy with phenotypes overlapping with EDMD, with dilated cardiomyopathy a common feature.

- Recent studies of the nuclear envelope are increasingly providing insights into its role in basic cellular functions, such as mechanotransduction and signal transduction, providing novel insights into the pathogenesis of EDMD.

- Recent advances obtained from studying the heart in mouse models of EDMD caused by LMNA mutations have identified potential therapeutic approaches.

- Ongoing research in small animal models should provide further insights into pathogenesis and other potentially novel therapies.

polypeptide 1. LMNA mutations different than those linked to muscular dystrophy and cardiomyopathy can cause partial lipodystrophy, peripheral neuropathy, and progeria [6].

Since these genetic discoveries, we know that the same mutations in $E M D$ and $L M N A$ can cause variable but overlapping phenotypes that differ in severity, onset, and rate of progression. In addition to 'classical' EDMD, skeletal muscle phenotypes caused by mutations in these genes include, limb-girdle, minimal to no skeletal muscle involvement, and - in the case of $L M N A$ - congenital muscular dystrophy $[7,8]$. Recently reported cases confirm this phenotypic variability $[9,10]$; however, the responsible mechanisms remain poorly understood.

\section{NUCLEAR ENVELOPE: THE FOCAL POINT OF EMERY -DREIFUSS MUSCULAR DYSTROPHY}

Emerin and A-type lamins, as well as nesprin-1, nesprin-2, SUN1, SUN2, LUMA, and lamina-associated polypeptide 1 are all localized to the nuclear envelope (Fig. 1). The nuclear envelope is, therefore, the cellular focal point for research on EDMD.

\section{Emerin}

Emerin is an integral protein that concentrates in the inner nuclear membrane of interphase cells. Emerin requires A-type lamins for its inner nuclear membrane localization [11]. A recent study has described the structural basis of the interaction between an immunoglobulin-like domain in A-type lamins and emerin [12"].

Emerin has been implicated in numerous cellular processes, including chromatin tethering, gene regulation, mitosis, nuclear assembly, and development signaling [13]. However, it is not essential in these fundamental cellular processes as loss of the protein in humans leads only to striated muscle disease. Either there is a high degree of redundancy at the tissue level or the role or emerin in some of these processes is only relevant in cell culture experiments.

\section{Lamins}

Nuclear lamins are intermediate filament proteins that polymerize to form the nuclear lamina [6]. In human, three genes encode nuclear lamins. LMNA encodes the A-type lamins, which in somatic cells are lamin $\mathrm{A}$ and lamin $\mathrm{C}$, arising by alternative RNA splicing. Two other genes, LMNB1 and LMNB2, respectively, encode the somatic cell B-type lamin B1 and lamin B2.

Recent progress has better defined the structure of the nuclear lamina in cultured cells. Cryoelectron tomography has shown that A-type and B-type lamins assemble into tetrameric filaments of $3.5 \mathrm{~nm}$ thickness, a structure different from the other canonical cytoskeletal intermediate filaments [14"']. More recent research using stochastic optical reconstruction microscopy has revealed that lamin B1 and lamin A/C form concentric but overlapping networks, with lamin B1 forming the outer ring located adjacent to the inner nuclear membrane [15"']. Extension of those structural studies to myoblasts and differentiated striated muscle cells, including those from affected patients, is likely to lead to a better understanding of EDMD.

\section{Nucleocytoplasmic connections: may the forces be with them}

Mechanisms of force transmission throughout the cell are particularly important in striated muscle. The Linker of Nucleoskeleton and Cytoskeleton (LINC) complex is composed of outer nuclear membrane Klarsicht, ANC-1, and Syne homology (KASH) as well as inner nuclear membrane Sad1 and UNC84 (SUN) proteins [16]. KASH and SUN proteins bind within the perinuclear space, the part of the endoplasmic reticulum lumen separating the inner and outer nuclear membranes. SUN proteins bind inside the nucleus to A-type lamins and also to emerin. KASH proteins, most of which are called nesprins in 


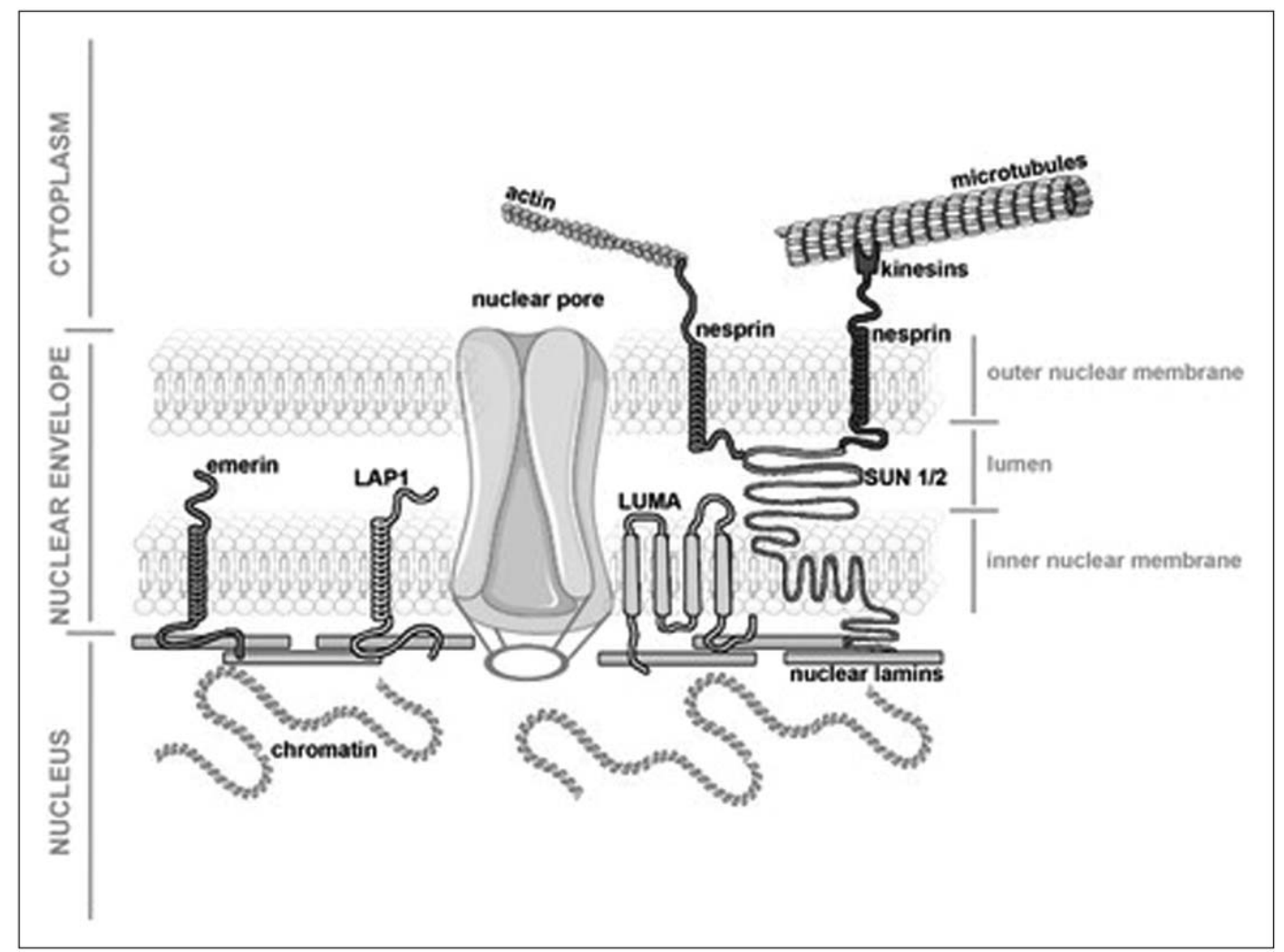

FIGURE 1. Schematic diagram of the nuclear envelope. Mutations in LMNA encoding A-type nuclear lamins, proteins of the nuclear lamina on the inner aspect of the inner nuclear membrane, cause autosomal dominant EDMD. Mutations in EMD encoding emerin, an integral protein of the inner nuclear membrane, cause X-linked EDMD. Mutations in genes encoding lamina-associated polypeptide 1 (LAP1), LUMA, SUN1, SUN2 have also been linked to EDMD-like phenotypes. Like emerin, LAP1 and LUMA are integral proteins of the inner nuclear membrane that bind to lamins. SUN1 and SUN2 are also integral inner nuclear membrane proteins that bind to lamins and to nesprins, integral proteins of the outer nuclear membrane, within the perinuclear space lume. The interactions between SUNs and nesprins form the LINC complex, which spans the nuclear envelope, connecting the nucleus to actin, to microtubules via motor proteins, such as kinesins, and to intermediate filaments via plectin (not shown) in the cytoplasm of somatic cells. EDMD, Emery-Dreifuss muscular dystrophy; LINC, Linker of Nucleoskeleton and Cytoskeleton.

mammals, bind to different cytoskeletal elements including actin, microtubules via dynein or kinesin1 , and intermediate filaments via plectin. LINC complexes, therefore, can transmit forces from throughout a cell, or even extracellularly, to the nucleus. Damage to any part of this complex force transmission system, which includes A-type lamins and emerin, may, therefore, make muscle fibers susceptible to damage by recurrent mechanical forces, causing muscular dystrophy and cardiomyopathy. Notably, EDMD-like phenotypes have been linked, albeit in some cases rather tentatively, to sequence variants of the human genes encoding nesprin-1, nesprin-2, SUN1, and SUN2 [17"].

Early research utilizing emerin-deficient mouse embryo fibroblasts showed that they have apparently normal nuclear mechanics but impaired expression of mechanosensitive genes in response to strain [18]. More recent experiments have shown that emerin is involved in the modulation of the spatial organization of chromosome territories in a manner that depends upon the stiffness of the substrate on which cells are cultured [19"]. This is consistent with previous research showing that emerin becomes tyrosine phosphorylated by applying forces directly to nesprin-1 using magnetic tweezers [20]. Emerin also organizes actin flow in polarizing fibroblasts, which provides the force, via nesprin-2G-SUN2-containg transmembrane actin-associated nuclear (TAN) lines, for nuclear movement necessary to establish polarity [21].

Loss of A-type lamins from cultured fibroblasts impairs nuclear mechanics and mechanotransduction, reflected by increased nuclear deformations, increased nuclear fragility, and attenuated expression of mechanosensitive genes, leading to impaired viability of mechanically strained cells [22]. Lamin A variants that cause EDMD are defective in anchoring 
TAN lines for nuclear movement in polarizing fibroblasts, indicating abnormal transmission of cytoplasmic forces to the nucleus [23]. A limitation of past research on the effects of lamins on cellular mechanics is that they were mostly carried out in fibroblasts rather than cell types affected in EDMD. Recently, atomic force microscopy and molecular modeling showed that neonatal rat ventricular cardiomyocytes have altered biomechanical properties when infected with adenoviral constructs directing expression of three cardiomyopathy-causing lamin A variants [24"]. These included disorganized cytoskeletal actin, alterations in nuclear elasticity (increased Young modulus), increased cell plasticity index, and lowered work of adhesion required to detach the tip of an atomic force microscopy cantilever from the cell membrane after indentation. The biomechanical abnormalities were reversed by treatment of the cells with a p38-alpha mitogen-activated protein kinase inhibitor, which also has beneficial effects on heart function in a mouse model of EDMD [25].

\section{THE HEART OF THE MATTER}

Neurologists often initially see patients with EDMD but virtually all will at some point see a cardiologist. Although the musculoskeletal symptoms can be debilitating, the dilated cardiomyopathy can be deadly. Patients are at risk for atrial fibrillation, complete heart block, lethal ventricular arrhythmias, and ultimately heart failure. Timing of placement of a pacemaker and/or defibrillator is critical yet not completely clear [8]. Many patients may ultimately require heart transplantation.

Much of the recent research on EDMD has been focused on the cardiomyopathy caused by LMNA mutations. Clinical research on this topic has been bolstered by collections of patients at several centers, although most so far has been retrospective. The availability of several genetically modified mice with Lmna mutations that develop cardiomyopathy has supported basic and translational research. Unfortunately, there have been few clinical studies on patients with EMD mutations and research has been limited by the fact that mice lacking emerin develop minimal symptoms.

\section{Recent clinical research on cardiomyopathy caused by LMNA mutations}

We have previously reviewed the prevalence and clinical features of cardiomyopathy caused by LMNA mutations [8]. A study from Norway published in 2018 , which found a $6.2 \%$ prevalence of LMNA mutation in patients referred for genetic testing for familial dilated cardiomyopathy, confirmed the high penetrance and relative severity, with frequently atrioventricular block, ventricular tachycardia, and a nearly $20 \%$ incidence of heart transplantation [26"]. Family screening diagnosed additional asymptomatic family members with LMNA mutations and follow-up revealed a 9\% annual incidence of a newly documented cardiac disease and $61 \%$ penetrance over 4.4 years of followup. Another study from Japan published in 2018 showed LMNA mutations in $24 \%$ of cases with familial dilated cardiomyopathy and, although the number identified was small, $69 \%$ had a family history of sudden cardiac death, $54 \%$ had sustained ventricular tachycardia, and 39\% underwent heart transplantation; most did not have evidence of left ventricular reverse remodeling (improvement in left ventricular function) after initiation of standard therapy for heart failure [27"]. LMNA mutations were also negative predictors for left ventricular reverse remodeling, which predicts a better outcome, in another group of patients with dilated cardiomyopathy after medical treatment [ $\left.28^{\prime}\right]$. These recent studies along with several previous ones show that cardiomyopathy caused by LMNA mutation is highly penetrant, severe, and refractory to standard heart failure therapy.

\section{Basic and translational research on cardiomyopathy caused by LMNA mutations}

Cell signaling abnormalities have been discovered in hearts of mouse models of cardiomyopathy caused by LMNA mutations. Several of these have been shown to contribute to pathogenesis including increased signaling by extracellular signal-regulated kinase 1 and kinase 2 (ERK1/2) and other mitogen-activated protein kinases, such as p38alpha, protein kinase $\mathrm{B} / \mathrm{mammalian}$ target of rapamycin complex 1 (AKT/mTOR), and transforming growth factor- $\beta$ [29]. Although it remains a mystery as to how alterations in A-type lamins activate these signal transduction pathways, pharmacological inhibition has beneficial effects on heart function in mice with cardiomyopathy and Lmna mutations. More recent research has implicated forkhead box $\mathrm{O}$ transcription factors and the DNA damage response/TP53 pathway in the pathogenesis of cardiomyopathy caused by LMNA mutations [30",31"]. Data from cardiomyocytes of human patients with LMNA mutations show that altered expression of TP53 and other genes correlates with the redistribution of lamina-associated domains of chromatin and changes in $\mathrm{CpG}$ methylation [32""]. 
A recent study in $L m n a^{\mathrm{H} 222 \mathrm{P} / \mathrm{H} 222 \mathrm{P}}$ mice, a model of cardiomyopathy in autosomal EDMD, has shed light on how ERK1/2 contributes to pathogenesis [33"']. Active, phosphorylated ERK1/2 directly binds to and catalyzes the phosphorylation of the cytoplasmic actin depolymerizing factor cofilin-1 on Thr25. This activates cofilin-1, which disassembles actin filaments. Phosphorylation of cofilin-1 on Thr25 along with ERK1/2 phosphorylation are also increased in human hearts from individuals with cardiomyopathy caused by LMNA mutations.

Recent studies have also expanded the number of pharmacological interventions that improve heart function in $L m n a^{\mathrm{H} 222 \mathrm{P} / \mathrm{H} 222 \mathrm{P}}$ mice in addition to the previously described beneficial effects of inhibitors of mitogen-activated protein kinases, mTOR, and the transforming growth factor- $\beta$ pathway [29]. Treatment with nicotinamide riboside, a pyridine-nucleoside form of vitamin B3, improved left ventricular structure and function, prolonged survival, and improved physical performance in a pilot study with small numbers of mice [34,35"]. Another study showed that $\mathrm{N}$-acetyl cysteine improves heart structure and function in Lmna $^{\mathrm{H} 222 \mathrm{P} / \mathrm{H} 222 \mathrm{P}}$ mice but no survival data were included [36]. Although these compounds are likely to be safe in human patients with cardiomyopathy caused by LMNA mutations, efficacy in mouse models of the disease should be compared directly to other drugs, such as pharmacological inhibition of ERK1/2 signaling [37].

\section{TOWARDS TREATMENTS: OF MICE AND MEN}

Mice such as those with the Lmna ${ }^{\mathrm{H} 222 \mathrm{P} / \mathrm{H} 222 \mathrm{P}}$ mutation fairly robustly mimic aspects of human autosomal dominant (although the mice are recessive) EDMD. However, larger animal models with cardiovascular and skeletal muscle physiology more similar to humans have been lacking. A recent report of LMNA knockout rabbits generated by CRISPS/Cas9 manipulation of zygotes may be a small step in the right direction; however, these animals are severely affected and die 22 days after birth [38]. More appropriate models would carry knock-in mutations, such as a recently described genetically engineered Yucatan minipig carrying a heterozygous LMNA mutation that causes Hutchinson-Gilford progeria syndrome [39"]. Nonetheless, given compelling data obtained in Lmna mutant mice for several therapeutic interventions, translation of some to human clinical trials would be appropriate. A preclinical proof of concept study in using an inhibitor of p38-alpha mitogen-activated protein kinase showed a significant benefit on dilated cardiomyopathy in
Lmna $^{\mathrm{H} 222 \mathrm{P} / \mathrm{H} 222 \mathrm{P}}$ mice [25]. This led to the launch of a Phase II, open-label, single arm clinical trial for patients with cardiomyopathy and LMNA mutations (ClinicalTrials.gov NCT02057341). Similar exploratory clinical trials could be considered based on other data obtained using this preclinical mouse model of EDMD caused by LMNA mutations.

Mice are highly resistant to depletion of emerin and germline Emd deletion causes minimal signs and symptoms $[40,41]$. Even those with combined germline deletion of both Lmna and Emd are born at expected Mendelian frequencies, although they have early growth retardation and a median survival of only approximately 3 weeks [42"]. This has limited translational research on X-linked EDMD. EMD mutations are almost always associated with lack of emerin expression $[43,44]$. This makes X-linked EDMD theoretically amenable to emerin replacement gene therapy and Emd null mice would be a suitable model to test protein expression, proper intracellular protein localization, and safety. Mice with combined germline deletion of Emd and selective skeletal muscle depletion of lamina-associated polypeptide 1 , which appears to compensate for loss of emerin in mice, may also serve as a reasonable model for testing preclinical therapeutic interventions [45].

Even with the best of preclinical research, better studies of human patients with EDMD are urgently needed. The dearth of prospective studies on the natural history of EDMD, especially the X-linked form caused by EMD mutations, has limited clinical guidelines for treatment. Similarly, the lack of large cohorts of patients with these rare genetic diseases makes randomized clinical trials difficult, although not insurmountable. This could be overcome by the establishment of shared, uniform patient registries, and more active involvement of patient organizations and support groups.

\section{CONCLUSION}

Recent research on EDMD is gradually being translated to clinical practice and possibly novel therapies. Larger, ideally prospective outcome studies with sufficient follow-up periods will better inform neurologists and cardiologists how to best care for patients. As EDMD is genetically heterogeneous, genetic testing and segregation based on the genetic cause - EMD, LMNA or other mutations - will be essential. Further basic research on the cell biology of the nuclear envelope along with more readily translatable studies utilizing animal models should identify novel target for therapeutic interventions. Such research has already begun being translated to patients. 


\section{Acknowledgements}

None.

\section{Financial support and sponsorship}

A.M. is supported by funds from the Association Française contre les Myopathies, the Institut National de la Santé et de la Recherche Médicale, Sorbonne Université and Agence Nationale de la Recherche (ANR-17CE17-0015-03). H.J.W. is supported by the NIH/NIAMS (R01AR048997).

\section{Conflicts of interest}

A.M. declares no potential conflict of interest. H.J.W. declares the following potential conflicts of interest: scientific advisory board and equity owner - AlloMek Therapeutics; scientific advisory board and consulting income from MNG Laboratories, consulting income from Eiger BioPharmaceuticals, sponsored research funding from Navitor Pharmaceuticals.

\section{REFERENCES AND RECOMMENDED READING}

Papers of particular interest, published within the annual period of review, have been highlighted as:

- of special interest

I. of outstanding interest

1. Emery $A E$, Dreifuss $F E$. Unusual type of benign $X$-linked muscular dystrophy. J Neurol Neurosurg Psychiatry 1966; 29:338-342.

2. Céstan R, LeJonne P. Une myopathie avec retractions familiales. Nouv Iconogr Salpetr 1902; 15:38-52.

3. Rowland LP, Fetell M, Olarte M, et al. Emery-Dreifuss muscular dystrophy. Ann Neurol 1979; 5:111-117.

4. Bione S, Maestrini E, Rivella S, et al. Identification of a novel X-linked gene responsible for Emery-Dreifuss muscular dystrophy. Nat Genet 1994; 8:323-327.

5. Bonne G, Di Barletta MR, Varnous $S$, et al. Mutations in the gene encoding lamin $\mathrm{A} / \mathrm{C}$ cause autosomal dominant Emery-Dreifuss muscular dystrophy. Nat Genet 1999; 21:285-288.

6. Worman HJ. Nuclear lamins and laminopathies. J Pathol 2012; 226 $316-325$.

7. Astejada MN, Goto K, Nagano A, et al. Emerinopathy and laminopathy clinical, pathological and molecular features of muscular dystrophy with nuclear envelopathy in Japan. Acta Myol 2007; 26:159-164.

8. Lu JT, Muchir A, Nagy PL, Worman HJ. LMNA cardiomyopathy: cell biology and genetics meet clinical medicine. Dis Model Mech 2011; 4:562-568.

9. Wang L, Zhang VW, Li S, et al. The clinical spectrum and genetic variability of limb-girdle muscular dystrophy in a cohort of Chinese patients. Orphanet J Rare Dis 2018; 13:133.

10. Zhou J, Li H, Li X, et al. A novel EMD mutation in a Chinese family with initial diagnosis of conduction cardiomyopathy. Brain Behav 2019; 9:e01167.

11. Sullivan $T$, Escalante-Alcalde $D$, Bhatt $H$, et al. Loss of A-type lamin expression compromises nuclear envelope integrity leading to muscular dystrophy. J Cell Biol 1999; 147:913-920.

12. Samson $C$, Petitalot $A$, Celli $F$, et al. Structural analysis of the ternary complex

- between lamin A/C, BAF and emerin identifies an interface disrupted in autosomal recessive progeroid diseases. Nucleic Acids Res 2018; 46:10460-10473.

The first structural description of the interaction between the immunoglobulin-like domain in A-type lamins and emerin. It revealed that this lamin domain both directly binds to self-assembled emerin and interacts with monomeric emerin LEM domain through the dimeric chromatin-associated barrier-to-autointegration factor protein. 13. Berk JM, Tifft KE, Wilson KL. The nuclear envelope LEM-domain protein emerin. Nucleus 2013; 4:298-314.

14. Turgay $Y$, Eibauer $M$, Goldman $A E$, et al. The molecular architecture of lamins In in somatic cells. Nature 2017; 543:261-264.

A landmark study utilizing cryo-electron tomography to obtain a detailed view of the organization of the lamin meshwork within the lamina. It showed that in vimentin-nul mouse embryonic fibroblasts, A-type and B-type lamins assemble into tetrameric filaments of $3.5 \mathrm{~nm}$ thickness. This structure is remarkably different from the canonical $10 \mathrm{~nm}$ thick filaments formed by cytoplasmic intermediate filament proteins.
15. Nmezi B, Xu J, Fu R, et al. Concentric organization of A- and B-type lamins

I. predicts their distinct roles in the spatial organization and stability of the nuclear lamina. Proc Natl Acad Sci U S A 2019; 116:4307-4315.

Using stochastic optical reconstruction microscopy, this study showed that lamin $B 1$ and lamin $A / C$ form concentric but overlapping networks, with lamin B1 forming the outer concentric ring located adjacent to the inner nuclear membrane.

16. Chang W, Worman HJ, Gundersen GG. Accessorizing and anchoring the LINC complex for multifunctionality. J Cell Biol 2015; 208:11-22.

17. Nagy PL, Worman HJ. Next-generation sequencing and mutational analysis:

- implications for genes encoding LINC complex proteins. Methods Mol Biol 2018; 1840:321-336.

An up-to-date outline of the most important and most commonly used materials and methods of next-generation sequencing, which can be used for high-throughput investigation of the LINC complex and to identify genetic variants in humans that may cause disease.

18. Lammerding J, Hsiao J, Schulze PC, et al. Abnormal nuclear shape and impaired mechanotransduction in emerin-deficient cells. J Cell Biol 2005; 170:781-791.

19. Pradhan R, Ranade D, Sengupta K. Emerin modulates spatial organization of

- chromosome territories in cells on softer matrices. Nucleic Acids Res 2018; 46:5561-5586

A study implicating emerin as a mechanosensor that modulates the spatial organization of chromosome territories in interphase nuclei. Chromosome territories of cells cultured on a softer surface demonstrated relocation of chromosome 1,18 and 19 territories into the nuclear interior. In these cells, emerin was phosphorylated on residue Tyr99 and expression of a phospho-deficient mutant (Y99F) led to retention of chromosome 18 and 19 territories at normal locations. 20. Guilluy C, Osborne LD, Van Landeghem L, et al. Isolated nuclei adapt to force and reveal a mechanotransduction pathway in the nucleus. Nat Cell Biol $2014 ; 16: 376-381$

21. Chang W, Folker ES, Worman HJ, Gundersen GG. Emerin organizes actin flow for nuclear movement and centrosome orientation in migrating fibroblasts. Mol Biol Cell 2013; 24:3869-3880.

22. Lammerding J, Schulze PC, Takahashi $T$, et al. Lamin A/C deficiency causes defective nuclear mechanics and mechanotransduction. J Clin Invest 2004; 113:370-378.

23. Folker ES, Östlund $C$, Luxton GW, et al. Lamin $A$ variants that cause striated muscle disease are defective in anchoring transmembrane actin-associated nuclear lines for nuclear movement. Proc Natl Acad Sci U S A 2011; 108:131-136.

24. Laurini $E$, Martinelli $V$, Lanzicher $T$, et al. Biomechanical defects and rescue of cardiomyocytes expressing pathologic nuclear lamins. Cardiovasc Res 2018; $114: 846-857$

This study utilized atomic force microscopy and molecular modeling to quantify biomechanical and structural defects induced by lamin A E161K, D192G and $\mathrm{N} 195 \mathrm{~K}$ variants in rat neonatal ventricular cardiomyocytes. Additionally, the biomechanical defects were rescued with a p38 inhibitor.

25. Muchir A, Wu W Choi JC et al. Abnormal $p 38 \alpha$ mitogen-activated protein kinase signaling in dilated cardiomyopathy caused by lamin $A / C$ gene mutation. Hum Mol Genet 2012; 21:4325-4333.

26. Hasselberg NE, Haland TF, Saberniak J, et al. Lamin A/C cardiomyopathy:

- young onset, high penetrance, and frequent need for heart transplantation. Eur Heart J 2018; 39:853-860.

A study from Norway examining the prevalence, cardiac penetrance and expressivity of LMNA mutations among familial dilated cardiomopathy in Norway. It demonstrated a relatively young onset, high penetrance and frequent need for heart transplantation.

27. Tobita $T$, Nomura $S$, Fujita $T$, et al. Genetic basis of cardiomyopathy and the - genotypes involved in prognosis and left ventricular reverse remodeling. Sci Rep 2018; 8:1998.

This study showed a relatively high incidence of $L M N A$ mutations among individuals with a history of familiar dilated cardiomyopathy in Japan. Although the number of affected individuals was small, it confirmed the relative severity of cardiac disease and showed low rate of left ventricular reverse remodeling after standard heart failure treatment.

28. Verdonschot JAJ, Hazebroek MR, Wang $P$, et al. Clinical phenotype and - genotype associations with improvement in left ventricular function in dilated cardiomyopathy. Circ Heart Fail 2018; 11 :e005220.

A study of left ventricular reverse remodeling in patients with dilated cardiomyopathy or hypokinetic nondilated cardiomyopathy that showed those with LMNA mutations are not likely to benefit remodeling after medical therapy.

29. Worman $\mathrm{HJ}$. Cell signaling abnormalities in cardiomyopathy caused by lamin A/C gene mutations. Biochem Soc Trans 2018; 46:37-42.

30. Auguste $G$, Gurha $P$, Lombardi $R$, et al. Suppression of activated FOXO

transcription factors in the heart prolongs survival in a mouse model of laminopathies. Circ Res 2018; 122:678-692.

Analysis of gene expression in hearts of $L m n a^{-/-}$mice identified upregulation of forkhead box $\mathrm{O}$ transcription factors and shRNA-mediated suppression prolonged survival.

31. Chen SN, Lombardi R, Karmouch J, et al. DNA damage response/TP53 pathway is

- activated and contributes to the pathogenesis of dilated cardiomyopathy associated with Imna (lamin A/C) mutations. Circ Res 2019; 124:856-873.

This study identified activation of the DNA damage response/TP53 pathway in hearts of transgenic mice with cardiomyocytes expression of the lamin A D300N variant and confirmed the results in hearts of human patients with LMNA mutations. Humans with the orthologous mutation to the mice; however, do not have a EDMD-like phenotype but rather 'atypical progeroid/Werner syndrome and nonsyndromic cardiac progeria'. 
32. Cheedipudi SM, Matkovich SJ, Coarfa C, et al. Genomic reorganization of

- lamin-associated domains in cardiac myocytes is associated with differential gene expression and DNA methylation in human dilated cardiomyopathy. Circ Res 2019; 124:1198-1213.

This study utilized human hearts to demonstrate redistribution of lamina-associated domains of chromatin in cardiomyocytes of patients with cardiomyopathy caused by LMNA mutations. These changes in chromatin organization correlated with alterations in gene expression, including TP53, and CpG methylation.

33. Chatzifrangkeskou $M$, Yadin $D$, Marais $T$, et al. Cofilin-1 phosphorylation

-1. catalyzed by ERK $1 / 2$ alters cardiac actin dynamics in dilated cardiomyopathy caused by lamin A/C gene mutation. Hum Mol Genet 2018; 27:3060-3078. This study provided evidence for how ERK $1 / 2$ hyperactivation contributes to heart dysfunction patients with cardiomyopathy caused by $L M N A$ mutation. It showed that activated ERK $1 / 2$ binds to and catalyzes the phosphorylation of the actin depolymerizing factor cofilin-1 on a specific amino acid residue. Cofilin-1 then becomes active and disassembles actin filaments.

34. Vignier N, Chatzifrangkeskou M, Morales Rodriguez B, et al. Rescue of biosynthesis of nicotinamide adenine dinucleotide protects the heart in cardiomyopathy caused by lamin A/C gene mutation. Hum Mol Genet 2018; 27:3870-3880.

35. Thomasson R, Vignier N, Peccate $\mathrm{C}$, et al. Alteration of performance in a

- mouse model of Emery-Dreifuss muscular dystrophy caused by A-type lamins gene mutation. Hum Mol Genet 2019; 28:2237-2244.

A demonstration that the $L m n a^{\mathrm{H} 222 \mathrm{P} / \mathrm{H} 222 \mathrm{P}}$ mouse model of autosomal EDMD mice displays a significant decrease in physical performance. In a pilot study, treatment with nicotinamide riboside improved physical performance parameters.

36. Rodriguez BM, Khouzami L, Decostre V, et al. N-acetyl cysteine alleviates oxidative stress and protects mice from dilated cardiomyopathy caused by mutations in nuclear A-type lamins gene. Hum Mol Genet 2018; 27:3353-3360.

37. Wu W, Chordia MD, Hart BP, et al. Macrocyclic MEK $1 / 2$ inhibitor with efficacy in a mouse model of cardiomyopathy caused by lamin A/C gene mutation. Bioorg Med Chem 2017; 25:1004-1013.
38. Sui T, Liu D, Liu T, et al. LMNA-mutated rabbits: a model of premature aging syndrome with muscular dystrophy and dilated cardiomyopathy. Aging Dis 2019; 10:102-115

39. Dorado B, Pløen GG, Barettino A, et al. Generation and characterization of a

- novel knockin minipig model of Hutchinson-Gilford progeria syndrome. Cell Discov 2019; 5:16.

This study describes the use of CRISPR/Cas9 gene editing to generate a minipig model of Hutchinson-Gilford progeria syndrome, which is caused by a unique mutation in $L M N A$, the same gene mutated in autosomal dominant EDMD.

40. Melcon G, Kozlov S, Cutler DA, et al. Loss of emerin at the nuclear envelope disrupts the $\mathrm{Rb} 1 / \mathrm{E} 2 \mathrm{~F}$ and $\mathrm{MyoD}$ pathways during muscle regeneration. Hum Mol Genet 2006; 15:637-651.

41. Ozawa R, Hayashi YK, Ogawa M, et al. Emerin-lacking mice show minimal motor and cardiac dysfunctions with nuclear-associated vacuoles. Am J Pathol 2006; 168:907-917.

42. Wang $\mathrm{Y}$, Shin JY, Nakanishi K, et al. Postnatal development of mice with

- combined genetic depletions of lamin $A / C$, emerin and lamina-associated polypeptide 1. Hum Mol Genet 2019. [Epub ahead of print]

A detailed characterization of the birth rates and postnatal development of mice with combined genetic deletions of the genes encoding A-type lamins, emerin and lamina-associated polypeptide 1 , three proteins that interact in the nuclear envelope; mutations in the orthologous human genes cause EDMD or EDMD-like phenotypes.

43. Nagano $A$, Koga $R$, Ogawa $M$, et al. Emerin deficiency at the nuclear membrane in patients with Emery-Dreifuss muscular dystrophy. Nat Genet $1996 ; 12: 254-259$

44. Manilal S, Nguyen TM, Sewry CA, Morris GE. The Emery-Dreifuss muscular dystrophy protein, emerin, is a nuclear membrane protein. Hum Mol Genet 1996; 5:801-808.

45. Shin JY, Méndez-López I, Wang Y, et al. Lamina-associated polypeptide-1 interacts with the muscular dystrophy protein emerin and is essential for skeletal muscle maintenance. Dev Cell 2013; 26:591-603. 